\title{
Los conversos según la documentación local de Cervera (1338-1501)
}

Josep M. ${ }^{a}$ LLOBET Portella *

\section{INTRODUCCIÓN}

Dentro de la extensa producción de estudios monográficos de contenido histórico debidos al profesor Dr. don Eloy Benito Ruano, el tema de los conversos ha sido tratado en varias ocasiones ${ }^{1}$. Por este motivo y como modesto homenaje a su persona tras un dilatado espacio de tiempo dedicado a la docencia universitaria y a la investigación ${ }^{2}$, quizá podría ser interesante aportar alguna información sobre dicho tema aun-

* Centro Asociado de la UNED en Cervera.

Algunos de dichos estudios: "El memorial contra los conversos del Bachiller Marcos García de Mora ("Marquillos de Mazarambroz")", Sefarad, XVII, 1957, págs. 314-351; "La Sentencia-Estatuto de Pero Sarmiento contra los conversos toledanos", Revista de la Universidad de Madrid, VI, 1957, págs. 277-306; «Del problema judío al problema converso», Simposio Toledano Judaico, II, Toledo, Centro Universitario de Toledo, 1972, págs. 5-28; Los origenes del problema converso, Barcelona, Ediciones El Albir, 1976.

2 El Dr. don Eloy Benito fue miembro del tribunal que juzgó mi tesis doctoral La "Taula de Canvi" de Cervera y su entorno socio-económico (1599-1715), Lleida, Instituto de Estudios llerdenses, 1985 y, posteriormente, aceptó participar en las Jornadas de Estudio «El món medieval a la Catalunya de Ponent», organizadas por el Centro Asociado de la Universidad Nacional de Educación a Distancia en Cervera. 
que sólo sea a partir de la documentación local que se ha conservado en la ciudad de Cervera ${ }^{3}$.

\section{CONVERSOS ANTERIORES AL AÑO 1391}

La información sobre conversos que nos ofrece la documentación local cervariense en el período anterior a las persecuciones del año 1391, es escasísima. Sólo conocemos un converso de Cervera y otro forastero que vino a predicar a la población.

Tenemos noticia del primero por medio de una anotación en el libro de Clavaria del año 1338, según el cual Bartomeu de Cervera, judío convertido al cristianismo, había recibido del Consejo municipal, como obsequio por su conversión, una gramalla que costó 12 sueldos ${ }^{4}$. Era costumbre que las autoridades cervarienses hicieran algún regalo a cada judío que se pasaba a la fe de Cristo, como lo demuestra un documento del año 1424 , en el que se precisa que los "paers" ${ }^{5}$ de la población habían entregado, en el año anterior, una cota de un valor de 23 sueldos a un judio que se había bautizado, «atenents que en semblans fets se acostume donar alcuna cosa per amor de Déu» ${ }^{6}$.

En cuanto al otro converso, la información nos llega a través del libro de Clavaria correspondiente al año 1339. Según una anotación, Pere

3 Si bien los judíos cervarienses han sido estudiados bajo diferentes aspectos, el tema de los conversos de la población no ha merecido, hasta el presente, una atención especial por parte de los historiadores. Los principales trabajos publicados son: A. DURAN I SANPERE: «Documents aljamiats de jueus catalans. Segle XV», Butlleti de la Biblioteca de Catalunya, V-VI, 1918-1919, págs. 132-148; Idem, Discursos llegits en la "Real Academia de Buenas Letras" de Barcelona en la solemne recepció pública de don Agusti Duran y Sanpere el día 20 d'abril de 1924. Referències documentals del call de juheus de Cervera, Barcelona, Real Academia de Buenas Letras, 1924. Estos dos trabajos se encuentran, revisados, en el capítulo "El Call dels jueus" de la obra del mismo autor titulada Llibre de Cervera, Tàrrega, Camps Calmet, 1972, págs. 343-390. Más información en A. DuRÁN I SANPERE, y M. SCHWAB: "Les juifs a Cervera et dans d'autres villes catalanes», Sefarad, XXXIV, 1974, págs. 79114.

4 Arxiu Històric Comarcal de Cervera, Fons Municipal, Clavaria, 1338, f. 49.

5 Los "paers" eran cuatro magistrados que, de forma anual y colegiada, ejercían el poder ejecutivo en el municipio cerveriense.

6 AHCC, FM, Consells, 1424, f. 63. 
Ferrández, converso que por honor a Dios predicaba la fe católica a los judíos, había recibido 20 sueldos del Consejo municipal ${ }^{7}$.

Dicho personaje, que en aquella ocasión había dirigido su palabra a la comunidad judía cervariense, quizá sería Pedro Fernández de Tudela, un converso que procedía de Tudela de Navarra y que, igual que su hermano Jimeno Pérez de Tudela, también converso, había obtenido del infante Pedro de Aragón, el 24 de agosto de 1333, una licencia para predicar a las aljamas de los judíos ${ }^{8}$.

\section{DE LAS PERSECUCIONES DE 1391 A LAS PREDICACIONES DE 1417}

Las persecuciones antijudaicas que en el año 1391 se extendieron por la Península lbérica ${ }^{9}$ también alcanzaron a los judíos cervarienses. Lo sucedido en dicha población está poco documentado, pero sabemos que hubo violencias que dieron lugar a varias disposiciones de las autoridades encaminadas a proteger a la comunidad judía ${ }^{10}$.

A partir de dicha fecha, la información documental sobre los conversos cervarienses o de otras poblaciones catalanas es mayor que durante el período anterior.

Según un documento notarial del 5 de enero de 1393, el converso Lluís de Junyent, que antes se llamaba Astruch Içach Adret y vivía en Cervera, vendió, desde Falset, varios bienes suyos al judío cervariense Içach de Quercí. En el mismo escrito consta el nombre del hijo de dicho vendedor, el también converso Mateu Bonjoch, anteriormente llamado Içach Astruch Adret ${ }^{11}$.

7 AHCC, FM, Clavaria, 1339, f. 10 v.

8 J. RIERA I SANS: "Les llicències reials per predicar als jueus $i$ als sarrainns (segles XIII-XIV)», Calls, 2, 1987, págs. 113-143. Sobre P. Fernández, pág. 122. 383-395.

9 Y. BAER: Historia de los judíos en la España cristiana, Madrid, Altalena, 1981, págs.

10. DuRán: Llibre de Cervera, págs. 351-353.

11 AHCC, Fons General, legajo Jueus, cuaderno. 
Otro escrito notarial nos informa de que, con fecha 18 de abril de 1395, Jaume Sabater, neophitus sive conversus, y su esposa Antonia, ambos de la villa de Cervera, recibieron cierta cantidad de dinero de Ramón Joan, también cervariense ${ }^{12}$. Dicho Sabater sería persona importante puesto que iniciado un proceso contra él por Ramóm Berenguer de Concabella y sus oficiales, el Concejo municipal, en el año 1403, acordó salir en su defensa, aunque con la condición de que todos los gastos corriesen a cargo del interesado ${ }^{13}$. Cuatro años después, Jaume Sabater era ciudadano de Barcelona si bien conservaba una casa en Cervera. La falta de pago de los impuestos locales, a los que estaba obligado por dicha propiedad, ocasionó la retención del importe del alquiler de la casa por parte de los "paers" cervarienses, hecho que provocó la protesta de los "consellers" de Barcelona y un intercambio de correspondencia entre las autoridades de ambas poblaciones ${ }^{14}$.

También un documento notarial, éste del año 1397, nos indica que el converso Francesc de la Brecerola, antes llamado Salamó Daví de Piera, era hijo y heredero universal del difunto Astengi Daví de Piera, judío de Cervera ${ }^{15}$. Dicho converso en el año 1430 aún vivía puesto que en aquella fecha vendió al judío Orabona una casa situada en el barrio judío, la cual había pertenecido a su padre ${ }^{16}$.

En el mes de junio de 1399, consta la existencia de otro converso cervariense, Joan de Queralt, que en dicha fecha nombró procurador suyo a Pere del Ginebret, también de Cervera ${ }^{17}$.

A principios del siglo $x \mathrm{~V}$, la cifra de los conversos que habitaban en Cervera quizá alcanzaría alguna importancia y muchos de ellos vivirian entre sus antiguos correligionarios. Así parece indicarlo las disposiciones del Consejo municipal que, reunido el 29 de abril de 1401, mandó que los conversos no continuasen residiendo entre los judíos, puesto que de esta forma cesarian «moltes errors que los converssos porien tenir e fer, stants ab los juheus ${ }^{18}$. La medida no debió resultar fácil de aplicar

\footnotetext{
AHCC, Fons Notarial, Cervera, n. ${ }^{\circ}$ 18, Pere de Salvanera, Llibre, 1395-1396, f. 99. AHCC, FM, Consells, 1403 , ff. 28 v. y 35.

AHCC, FM, Registre de Lletres, 1406-1408, ff. 58 v. y 70.

AHCC, FN, Cervera, n. ${ }^{\circ} 21$, Jaume Forner, Llibre, 1397-1400, f. 3.

$\mathrm{AHCC}, \mathrm{FM}$, Consells, 1430, f. $86 \mathrm{v}$.

AHCC, FN, Cervera, n. ${ }^{\circ} 22$, Pere Solanelles, Manual, 1399-1401, f. $110 \mathrm{v}$.

AHCC, FM, Consells, 1401, f. 49.
} 
puesto que tres meses después el Consejo confía a los «paers» la ejecución de todo este asunto ${ }^{19}$.

En el mes de enero del año siguiente, se continúa insistiendo sobre la separación de los conversos, aunque en esta ocasión el escribano equivoca la palabra y, en vez de judíos, pone cristianos ${ }^{20}$.

El acta de una reunión del Consejo municipal, celebrada el 18 de diciembre de 1406, nos facilita el nombre de otro converso cervariense: Francesc Miró, que anteriormente se llamaba Bonjuhà Astruch y era propietario de una casa en el barrio judío ${ }^{21}$.

La documentación cervariense, además de los conversos de la población, se hace eco de varios neófitos de otros lugares de Cataluña.

Así, con fecha 23 de noviembre de 1399, Andreu Ferran, de Tárrega, neofitus sive conversus, junto con otras personas, reconocía una deuda a Constança, viuda de Arnau Serradell ${ }^{22}$.

Dos años después, dos conversos de la villa de Montblanc que se habían hecho vecinos de Cervera, solicitaban un aplazamiento en el cambio de residencia con el fin de poder solucionar, ellos y otros compañeros conversos de aquella población, algunos problemas económicos ${ }^{23}$.

En el mes de septiembre de 1404, Joan Palau, converso de Perpiñán, reconocía, entre otras cosas, haber recibido como primera paga 11 sueldos de Guillem Savila, sastre de Cervera ${ }^{24}$.

Finalmente, según una anotación del año 1408, otro converso, Miquel Mulner, presentó una reclamación en los tribunales de Tárrega contra los herederos del difunto Bertran dels Archs, vecino de Cervera ${ }^{25}$.

\footnotetext{
19 AHCC, FM, Consel/s, 1401, f. 74.

20 AHCC, FM, Consells, 1402, f. 21.

21 AHCC, FM, Consells, 1406, f. 89 v.

22 AHCC, FN, Cervera, n. ${ }^{\circ}$ 22, Pere de Solanelles, Manual, 1399-1401, f. 135.

$23 \mathrm{AHCC}, \mathrm{FM}$, Consells, 1401, f. 125.

24 AHCC, FN, Cervera, n.0 18, Pere de Salvanera, Manual, 1403-1404, f. 226.

25 AHCC, FM, Clavaria, 1408, f. 23.
} 


\section{DE LAS PREDICACIONES DE 1417 A LAS DEL AÑO 1420}

Una anotación del año 1417 nos informa que en aquella fecha un maestro llamado Gil Benet predicó en la iglesia de Santa María de Cervera durante tres días. Sus sermones fueron dirigidos tanto a los judíos como a los cristianos y recibió 3 florines del Consejo municipal en concepto de limosna ${ }^{26}$.

A partir de esta fecha y al menos durante la primera mitad del siglo $x v$, en varias ocasiones tuvieron lugar predicaciones en Cervera, como veremos más adelante.

Quizá el movimiento espiritual que provocó entre los cristianos la llamada disputa de Tortosa ${ }^{27}$, conjunto de sesiones de discusión doctrinal entre judíos y cristianos que se desarrollaron durante los años 1413 y 1414 , no fuera ajeno a los afanes redentoristas mostrados por los predicadores. Sea como fuere, poco después de los sermones del año 1417 van apareciendo varios nombres de conversos en los documentos cervarienses.

En dos anotaciones del año 1418, se habla de Manuel de Ribelles, neófito residente en Cervera, y de su esposa Durona, que era judiá ${ }^{28}$. Otra, fechada en 1421, precisa que Manuel de Ribelles se llamaba, cuando era judío, Içach Samuel Baruch ${ }^{29}$. Una tercera, que corresponde a 1429, sitúa a dicho converso, en aquel año, en la villa de Agramunt ${ }^{30}$.

Según un documento de 1418, un yerno de Manuel de Ribelles se llamaba Lluís de Sentgil y era, también, converso y vecino de Cervera ${ }^{31}$. Una anotación del año 1421 permite saber de este último que su esposa se llamaba Bonafilla - nombre judío- y su madre Regino - también el nombre es judio, pero consta que se había hecho cristiana- ${ }^{32}$. Efectivamente, en un inventario del año 1422 aparece un tal Marquesa como

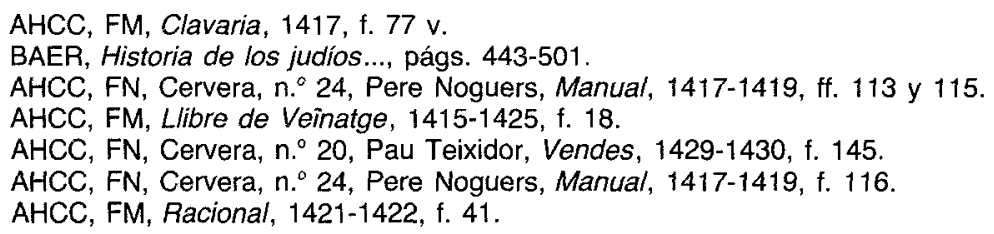


madre del converso Lluís de Sentgil y esposa de Joan Gilabert, de Cardona ${ }^{33}$.

La misma anotación de año 1421, que hemos comentado, nos informa de que un judío cervariense llamado Samuel de Piera se había convertido al cristianismo, pero no da su nuevo nombre.

El día 19 de marzo de 1420, fue aceptada como vecina de Cervera una judía llamada Goyo que procedía de la villa de Ponts ${ }^{34}$. Había sido esposa del converso Francesc de Tovia, antes llamado Bonet Salamies de Llunel, y tenía tres hijos: Samuel de Llunell, Salamies de Llunell -vecinos de Cervera en el año $1416{ }^{35}$ - y Cresques de Llunell, que en el año 1427 figura como difunto ${ }^{36}$.

Otros conversos que aparecen en la documentación cervariense del período comprendido entre 1417 y 1420 son los siguientes:

En el mismo año 1417 consta que un converso de Tárrega, llamado Ramón Sisquer, se había trasladado a Cervera para pedir el pago de una cantidad de dinero que esta población debía a Francesc Ferrer ${ }^{37}$.

Del año 1418 tenemos un interesante documento. Es un acta notarial que certifica la negativa de la esposa de un converso a regresar con él a Gerona ${ }^{38}$. En efecto, el 17 de febrero de aquel año Pere Armengou, neófito de la ciudad de Gerona se trasladó personalmente a la villa de Cervera y en el lugar llamado «les graus de Sant Cristòfol», cerca del horno de un tal Vidal, encontró a su mujer y entre ellos tuvo lugar el siguiente diálogo:

- Na Goyo, ma muller sou, jo us he a sperar un any despuys que.m só fet christià, segons la ordinatió del papa e licèntia que n'e. Jo,

33 DURÁN Y SCHWAB: «Les juifs a Cervera...», pág. 96.

34 AHCC, FM, Llibre de Veinatge, 1415-1425, f. 44 v. Sobre la entrada en vecindad de ella y de otras personas de Ponts: J. M. ${ }^{3}$ LLOBET I PONTELLA: El veinatge de Ponts amb Cervera durant la primera meitat del segle $X V$, en prensa.

35 AHCC, FM, Llibre de Veinatge, 1415-1425, f. 20.

36 AHCC, FN, Cervera, n. ${ }^{\circ}$ 28, Jaume Gualter, Manual, 1427, f. 48.

37 AHCC, FM, Clavaria, 1417, f. 74 v. Publicado en : J. M. ${ }^{a}$ LlOBET I PORTELLA: Algunes noticies sobre els jueus de Tàrrega (1303-1486), Lleida, Institut d'Estudis Ilerdencs, 1984, pág. 14.

38 AHCC, FN, Cervera, n. ${ }^{\circ}$ 24, Pere Noguers, Manual, 1417-1419, f. 36. 
axí com a aquella qui sou ma muller, vós he menada de Girona aci he us he feta la companyia que devia. Ara jo me'n vull tornar a Girona en ço del me. E axí prech vós que.n en tornem car per ma fe jo us faré la companya bona a leal que marit deu fer a muller e axi anem cat tantost mich vuyll partir.

- Ver és que jo só vostra mullé mas no me'n vuyl anar car ací me vuyll star.

- Jo us requir que us n'aneu ab mi, en altre manera jo protest contra vós de adquisició de vostre dot, spoli e altres béns o de tots altres dret a mi licis de protestar e requirir e pero ço com me sou desobedient requir vós, notari, que u continueu e que me'n façau carta.

- Vos porets fer que us volrets que yo no y hiré ne vuy exir de Cervera ans me apell de ço que.m dicís.

Otro documento notarial, fechado once días después del anterior, incluye el nombre de un converso de la villa de Perpiñán: Pere Bonanat ${ }^{39}$.

Un tercer documento, también notarial y con la misma fecha que el anterior, nos informa de que un converso de Agramunt, que se llamaba Francesc Queralt, tenía una hermana judia, de nombre Gemila, que había sido esposa del difundo Abrae Baruc ${ }^{40}$.

El último documento de este período, fechado el 12 de septiembre de 1418, nos permite saber que Antoni Girgós, neófito residente en la villa de Castellón de Ampurias, anteriormente se llamaba Içach Adret y había sido yerno de Alí Salamó, judio de Gerona que se trasladó a vivir a Cervera ${ }^{41}$.

39 AHCC, FN, Cervera n. ${ }^{\circ}$ 24, Pere Noguers, Manual, 1417-1419, f. 43.

40 AHCC, FN, Cervera, n. ${ }^{\circ}$ 24, Pere Noguers, Manual, 1417-1419, f. 43.

41 AHCC, FN, Cervera, n. ${ }^{\circ}$ 24, Pere Noguers, Manual, 1417-1419, f. $108 \mathrm{v}$. 


\section{DE LAS PREDICACIONES DE 1420 A LA EXPUSIÓN DE LOS JUDÍOS}

En el mes de octubre de 1420, el Concejo municipal de Cervera se reunió para tratar de las graves acusaciones que un fraile predicador, que había dirigido la palabra a los cervarienses durante varios días, había hecho a las autoridades locales, a quienes responsabilizaba de los "greus peccats e abominacions qui.s fan continuament en aquesta vila". Entre los pecados denunciados, se hacía mención de que los judíos, enemichs de nostre senyor Déus Ihesu Christ, no llevaban la «roda» o señal exterior, vivian entre los cristianos y les vendian la carne que ellos reusaban comer ${ }^{42}$.

El Consejo cervariense, "per descàrrech de ses conciències", elaboró una serie de ordenanzas que, entre otras cosas, disponía la obligación de que los judíos llevasen la señal que los identificaba, viviesen separados de los cristianos y consumiesen la carne de los animales que sacrificaran, la cual no podía ser vendida a los cristianos.

No consta que el fraile predicador dirigiese algunos de sus sermones a los judíos ni que éstos asistiesen a las predicaciones. En cambio, sabemos que en el año 1431 se mandó a los judíos cervarienses que estuviesen presentes en el sermón del maestro Lucas $^{43}$ y que, aquel mismo año, el maestro Gilabert, que era converso, recibió del Consejo municipal 5 sueldos por un sermón en el cual asistieron todos los judíos de la villa ${ }^{44}$. No queda claro si fueron realmente dos sermones o el maestro Lucas y el maestro Gilabert eran la misma persona.

En el año 1436, de nuevo se predica en Cervera. Esta vez por un fraile de la Merced que también se queja de que los judíos habiten entre los cristianos ${ }^{45}$. En esta ocasión, tampoco consta que se dirigiera concretamente a los judíos. Parece ser que las predicaciones a dicha comunidad se reservaban, generalmente, a los conversos; quizá así resultaban más eficaces.

\footnotetext{
42 AHCC, FM, Consells, 1420, f. 56 v. Publicado por DuRÁn: Discursos..., págs. 57-60.

43 AHCC, FM, Clavaria, 1431, f. 103.

44 AHCC, FM, Clavaria, 1431, f. 103 v.

45 AHCC, FM, Consells, 1436, f. $91 \mathrm{v}$.
} 
A pesar de ello, en este período no aparece un gran número de conversos en la documentación local. Los que hemos localizado son los siguientes:

En un documento notarial del año 1421, Francesc d'Oluja, converso de Cervera, reconoce haber recibido 20 florines de Llorenç Estalrich del lugar de Conesa ${ }^{46}$.

Ya hemos dicho anteriormente que, en el año 1423, el Consejo municipal cervariense regaló una cota a un judío que se bautizó, sin que sepamos su nombre.

Otro converso oriundo de Cervera era Joan Gràcia, miembro de la Orden militar de San Jaime, quien, con fecha 13 de mayo de 1456, confiesa haber recibido 200 florines de su padre, Içach Gràcia, judío cervariense ${ }^{47}$.

A principios del año 1428, se hace mención de Ferran de Fonellet, converso de la villa de Perpiñán, quien reconoce tener en depósito de Antoni Calbó de la ciudad de Barcelona, 70 sueldos ${ }^{48}$.

De Santa Coloma de Queralt, tenemos constancia de dos conversos, uno conocido por Ramonet, según una anotación del año $1430^{49}$, y otro llamado Gabriel de Ribelles, que aparece en un documento notarial de $1433^{50}$.

Un neófito de procedencia desconocida es Ramón Gilabert, quien, en el año 1440, reconoce tener en depósito de Gerard Tallada de la villa de Cervera, cierta cantidad de dinero ${ }^{51}$.

Dentro de este mismo período, fue expedida una carta con fecha 7 de diciembre de 1481 por el infante Enrique de Aragón, según la cual una judía ya fallecida, llamada Regino, se había hecho cristiana con el nombre de lolanda y había sido esposa primero del judío cervariense

\footnotetext{
46 AHCC, FN, Cervera, n. 29, Ramon Solsona, Manual, 1421, f. 162 bis v. AHCC, FN, Cervera, n. ${ }^{\circ} 35$, Joan d'Albalat, Manual, 1456, ff. 81 y 82.

AHCC, FN, Cervera, n. ${ }^{\circ} 32$, Jaume d'Agramuntell, Llibre, 1428-1431, f. 2. AHCC, FM, Consells, 1430, f. 85.

AHCC, FN, Cervera, n. ${ }^{\circ}$ 20, Pau Teixidor, Manual, 1432-1433, f. $147 \mathrm{v}$.

AHCC, FN, Cervera, n. ${ }^{\circ} 32$, Jaume d'Agramuntell, Manual, 1439-1449, f. 10.
} 
Jacob de Quercí, en aquel momento habitante de Anglesola o Tárrega, y después de Bernardí Desplà, quien residía en Barcelona ${ }^{52}$. Dicha judía era hija de un judío de Gerona, también difunto, llamado Salamó de Tolosa.

\section{LOS CONVERSOS DESPUES DE LA EXPULSIÓN DE LOS JUDíOS}

A finales de abril y principios de mayo de 1492 se hizo público el edicto que ordenaba la expulsión de los judíos de todos los territorios de las coronas castellana y aragonesa ${ }^{53}$. Antes de su salida era preciso que saldaran sus deudas y este aspecto fue un tema debatido en varias reuniones del Consejo municipal de Cervera a partir del conocimiento que se tuvo de aquella orden. ${ }^{54}$.

Después de la expulsión de los judíos cervarienses, las menciones de conversos en la documentación local van escaseando hasta desaparecer totalmente en su forma explícita.

Una de las familias judías notables que se quedó en Cervera fue la de los Quercí ${ }^{55}$, que tomó el nombre de Pau. Algunos de sus miembros fueron los siguientes: Jaume de Pau, médico, que antes se llamó lçach Jacob de Quercí ${ }^{56}$, Manuel Joan de Pau, también médico ${ }^{57}$, y Francí de Pau, mercader ${ }^{58}$.

52 AHCC, FG, legajo Jueus.

53 BAER, Historia de los judíos..., pág. 646.

54 AHCC, FM, Consells, 1492.

55 DURÁN: (Llibre de Cervera, pág. 382) ya intuyó que se convirtieron con el nombre de Pau, aunque no dispusiera de un documento explícito como el que hemos localizado nosotros.

56 AHCC, FM, Consells, 1492, ff. 34 v. y 65; FN, Cervera, n. ${ }^{\circ}$ 52, Llorenç Serra, Manual, 1492-1493, f. 21 v.; n. ${ }^{\circ}$ 48, Berenguer Gassó, Llibre, 1495-1498, f. 52; FM, Consells, 1501 , f. $17 \mathrm{v}$.

${ }_{57}$ AHCC, FN, Cervera, n. ${ }^{\circ}$ 52, Llorenç Serra, Manual, 1492-1493, f. 19; FM, Clavaria, 1495, f. 16 v.; FN, Cervera, n. ${ }^{\circ} 48$, Berenguer Gassó, Liibre, 1495-1498, f. 92.

${ }_{58}$ AHCC, FM, Clavaria, 1495, f. 16 v.; FN, Cervera, n. ${ }^{\circ}$ 48, Berenguer Gassó, Llibre, $1495-1498$, f. 62 v. 
Otra familia importante, también de médicos judíos, fue la de los Cavaller ${ }^{59}$. Uno de ellos, Samuel Cavaller se marchó de Cervera antes de la expulsión ${ }^{60}$. Otro, que se quedó en la villa se cristianizó con el nombre de Joan de Cardona ${ }^{61}$.

Finalmente, en un documento notarial expedido en el año 1497, aparece como testigo Salvador Boix, neófito de Cervera ${ }^{62}$. Con su nombre cerramos esta lista de conversos que nos ha ofrecido la documentación local cervariense.

\section{CONCLUSIONES}

A partir de todo lo que llevamos dicho en las páginas anteriores, podemos establecer las siguientes conclusiones:

- La información sobre conversos anterior a las persecuciones del año 1391 es muy escasa. Si bien los conversos, en aquellas fechas, serían pocos, la pérdida de buena parte de las fuentes documentales puede contribuir a dicha falta de información.

- Entre los años 1391 y 1417, varios nombres de conversos van apareciendo en las anotaciones de la documentación local. Sin embargo, poco más de una decena, entre cervarienses y forasteros, en un período de veintiséis años no parece una cifra demasiado elevada. Claro que las disposiciones de principios del siglo $\mathrm{xV}$, que ordenan que los conversos no continuen viviendo entre los judíos, parecen indicar que el número de conversos que habitaban en Cervera era muy superior a los que nos ofrece la documentación del período.

59 Puede verse: DuRÁN: Llibre de Cervera, págs. 379 y 380; BaER: Historia de los judios..., pág. 610.

${ }_{60}$ AHCC, FN, Cervera, n. ${ }^{\circ} 55$, Antoni Romeu, Manual, 1489-1490, documento en la bolsa de la cubierta.

${ }^{61}$ AHCC, FN, Cervera, n. ${ }^{\circ}$ 55, Antoni Romeu, Manual, 1495-1497, documento en la bolsa de la cubierta; $n .^{\circ} 48$, Berenguer Gassó, Llibre, 1495-1498, f. 92.

${ }^{62}$ AHCC, FN, Cervera, n. ${ }^{\circ} 48$, Berenguer Gassó, Llibre, 1495-1498, f. 62. 
- En los tres años que median entre 1417 y 1420, aparece en la documentación cervariense un número de conversos parecido al del período anterior de veintiséis años. No parece aventurado suponer que ello pueda estar relacionado con el movimiento de conversiones que generó la disputa de Tortosa.

- Entre 1420 y 1492, fecha de la expulsión de los judíos, las fuentes documentales locales no ofrecen un número elevado de conversos. $Y$ esto es más acusado en la segunda mitad del siglo XV. Quizá las comunidades judías, especialmente después de la guerra que se sostuvo en Cataluña contra Juan II de Aragón, se hallaban ya en una situación de decadencia.

- Después del año 1492 el rastro de los conversos se pierde rápidamente. La mención expresa de su condición, pronto es evitada en la redacción de los documentos. Sólo, de vez en cuando, aparece, después del apellido cristiano, el antiguo nombre judío precedido de la palabra alias.

- A partir de principios del siglo XVI son escasísimos los elementos que permiten identificar un converso en la documentación local. Puede decirse que, al menos en su aspecto público, la conversión de los judíos que permanecieron en la población había sido tan rápida como efectiva. 



\section{APÉNDICE}

Indice alfabético de los nombres de los conversos. Se incluye el nombre judío cuando se conoce. También se indican los años en que el nombre cristiano se halla documentado.

ARMEngou, Pere, 1418.

BOIX, Salvador, 1497.

BONANAT, Pere, 1418.

BONJOCH, Mateu (Içach Astruch Adret), 1393.

BRECEROLA, Francesc de la (Salamó Daví de Piera), 1397-1430.

CARDONA, Joan de (? Cavaller), 1495-1497.

CERVERA, Bartomeu de, 1338.

FERRAN, Andreu, 1399.

FERRÁNDEZ, Pere, 1339.

FONELLET, Ferran de, 1428.

GILABERT, 1431.

GILABERT, Ramón, 1440.

GIRGOS, Antoni (Içach Adret), 1418.

GRÀCIA, Joan, 1456.

JUNYENT, Lluís de (Astruch Içach Adred), 1393.

MIRÓ, Francesc (Bonjuhà Astruch), 1406.

MULNER, Miquel, 1408. 
OLUJA, Francesc d', 1421.

PALAU, Joan, 1404.

PAU, Franci de (? de Quercí), 1495-1497.

PAU, Jaume de (Içach Jacob de Quercí), 1492-1501.

PAU, Manuel Joan de (? de Quercí), 1493-1497.

QUERALT, Francesc, 1418.

QUERALT, Joan de, 1399.

RAMONET, 1430.

RIBELLES, Gabriel de, 1433.

RIBELLES, Manuel de (Içach Samuel Baruch), 1418-1429.

SABATER, Jaume, 1395-1407.

SENTGIL, Lluís de, 1418-1422.

SISQUER, Ramon, 1417.

TOVIA, Francesc de (Bonet Salamies de Llunell), 1420-1427. 